Article

\title{
Liquid Chromatographic Resolution of Fendiline and Its Analogues on a Chiral Stationary Phase Based on (+)-(18-Crown-6)-2,3,11,12-tetracarboxylic Acid
}

\section{Ga Ram Lee and Myung Ho Hyun*}

Department of Chemistry and Chemistry Institute for Functional Materials, Pusan National University, Busan 690-735, Korea; E-Mail: 1gr8969@pusan.ac.kr

* Author to whom correspondence should be addressed; E-Mail: mhhyun@pusan.ac.kr; Tel.: +82-51-510-2245; Fax: +82-51-516-7421.

External Editor: Stig Allenmark

Received: 4 November 2014; in revised form: 5 December 2014 / Accepted: 11 December 2014 / Published: 19 December 2014

\begin{abstract}
Fendiline, an effective anti-anginal drug for the treatment of coronary heart diseases, and its sixteen analogues were resolved on a CSP based on (+)-(18-crown-6)2,3,11,12-tetracarboxylic acid. Fendiline was resolved quite well with the separation factor $(\alpha)$ of 1.25 and resolution ( $\mathrm{RS}_{\mathrm{S}}$ ) of 1.55 when a mobile phase consisting of methanolacetonitrile-trifluoroacetic acid-triethylamine at a ratio of $80 / 20 / 0.1 / 0.5(\mathrm{v} / \mathrm{v} / \mathrm{v} / \mathrm{v})$ was used. The comparison of the chromatographic behaviors for the resolution of fendiline and its analogues indicated that the 3,3-diphenylpropyl group bonded to the secondary amino group of fendiline is important in the chiral recognition and the difference in the steric bulkiness between the phenyl group and the methyl group at the chiral center of fendiline is also important in the chiral recognition.
\end{abstract}

Keywords: chiral stationary phase; enantiomer separation; liquid chromatography; fendiline

\section{Introduction}

Fendiline (Figure 1) is an effective anti-anginal drug for the treatment of coronary heart diseases. The anti-anginal effects of fendiline have been known to be attributed largely to its dilatory effects on small blood vessels [1]. Fendiline is a chiral drug containing one chiral center. Two enantiomers of 
chiral drugs have been known often to show different biological activities including pharmacology, toxicology and pharmacokinetics [2]. Two enantiomers of fendiline also have the good chance of showing different biological activities in our body. Actually, the $(R)$-enantiomer of fendiline was found to show a more potent vasodilatory effect than the $(S)$-enantiomer [3]. In this instance, the exact determination of the enantiomeric composition of fendiline is very important. Among various methods, liquid chromatographic chiral stationary phase (CSP) method has been known to be one of the most accurate, convenient and economic means for the determination of enantiomeric composition of chiral drugs [4]. Various liquid chromatographic CSPs have been applied to the determination of enantiomeric composition of chiral compounds [5,6]. For example, CSPs based on polysaccharide derivatives [7], cyclodextrines [8], macrocyclic antibiotics [9,10], cyclofrutanes [11], chiral crown ethers [11-14] and other small molecules [15] were quite successful for the resolution of racemic compounds. However, liquid chromatographic enantioseparation of fendiline on CSPs is quite rare. Only a CSP based on $\alpha_{1}$-acid glycoprotein (AGP) was used for the enantioseparation of fendiline by liquid chromatography [16].

Figure 1. Structures of fendiline and chiral stationary phase (CSP) 1.

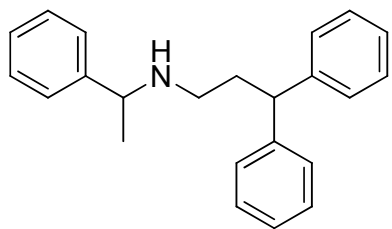

Fendiline

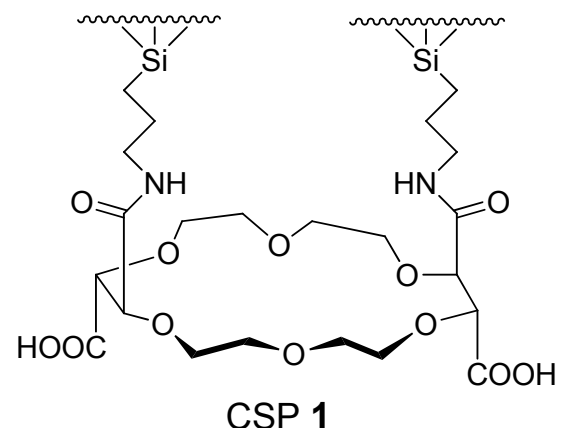

CSP 1

CSPs based on chiral crown ethers were very successful in the determination of enantiomeric composition of chiral compounds containing a primary amino group [12,17-19]. While CSPs based on chiral crown ethers have been known to be effective for the resolution of racemic compounds containing a primary amino group through the enantioselective complexation of the primary ammonium group $\left(\mathrm{R}_{-} \mathrm{NH}_{3}{ }^{+}\right)$of analytes inside the cavity of the crown ether ring of the stationary phase [12,17-19], interestingly CSP 1 (Figure 1) based on (+)-(18-crown-6)-2,3,11,12-tetracarboxylic acid was found to be also quite successful in the resolution of racemic compounds containing a secondary amino group such as $\beta$-blockers [20], flecainide analogues [21], isoquinolines [22] and rasagiline analogues [23]. Fendiline also contains a secondary amino group and, in this instance, it is expected to be resolved on CSP 1. In this study, we wish to report the resolution of fendiline and its analogues (2-17) shown in Figure 2 on CSP 1.

\section{Results and Discussion}

The structural characteristics required for the resolution of fendiline on CSP 1 might be elucidated by comparing the chromatographic resolution behaviors for the resolution of fendiline and its analogues. Consequently, various types of fendiline analogues shown in Figure 2 were prepared. In order to see the importance of the 3,3-diphenylpropyl group of fendiline, analogues (2-6) containing simple straight chain alkyl group, sterically bulky alkyl group or phenylalkyl group at the nitrogen of the secondary 
amino group were prepared. Analogue (7) containing a propyl group instead of the methyl group at the chiral center of fendiline was also prepared to see the effect of the methyl group on the chiral resolution. Analogues (8-12) containing ortho-substituted phenyl or 1-naphthyl group and analogues (13-17) containing para-substituted phenyl or 2-naphthyl group instead of the simple phenyl group were prepared for the purpose of elucidating the role of the phenyl group at the chiral center of fendiline in the chiral recognition.

Figure 2. Structures of fendiline analogues (2-17).<smiles>CCCCNC(C)c1ccccc1</smiles><smiles>CC(NCCc1ccccc1)c1ccccc1</smiles><smiles>CC(NCCC(c1ccccc1)c1ccccc1)c1ccccc1Br</smiles><smiles>CC(NCCC(c1ccccc1)c1ccccc1)c1ccc(F)cc1</smiles><smiles>CC(C)CNC(C)c1ccccc1</smiles><smiles>CCCC(NCCC(c1ccccc1)c1ccccc1)c1ccccc1</smiles><smiles>COc1ccccc1C(C)NCCC(c1ccccc1)c1ccccc1</smiles><smiles>CC(NCCC(c1ccccc1)c1ccccc1)c1ccc(Br)cc1</smiles><smiles>CC(NCCC(C)(C)C)c1ccccc1</smiles><smiles>Cc1ccccc1C(C)NCCC(c1ccccc1)c1ccccc1</smiles><smiles>CC(NCCC(c1ccccc1)c1ccccc1)c1cccc2ccccc12</smiles><smiles>COc1ccc(C([18F])NCCC(c2ccccc2)c2ccccc2)cc1</smiles><smiles>CCCCCCCCNC(C)c1ccccc1</smiles><smiles>CC(NCCC(c1ccccc1)c1ccccc1)c1ccccc1F</smiles><smiles>Cc1ccc(C(C)NCCC(c2ccccc2)c2ccccc2)cc1</smiles><smiles>[3H]C(NCCC(c1ccccc1)c1ccccc1)c1ccc2ccccc2c1</smiles>

For the resolution of secondary amino compounds on CSP 1, mobile phase composition has been known to be very important. For the resolution of secondary amino alcohols related to $\beta$-blockers on CSP 1, a mixture of ethanol-acetonitrile-trifluoroacetic acid-triethylamine (20/80/0.1/0.5, v/v/v/v) was successfully used as a mobile phase [20]. However, for the resolution of flecainide and its analogues on CSP 1, a different mobile phase consisting of methanol-acetonitrile-trifluoroacetic acidtriethylamine $(80 / 20 / 0.1 / 0.3, \mathrm{v} / \mathrm{v} / \mathrm{v} / \mathrm{v})$ was most widely applied [21]. For the resolution of rasagiline and analogues on CSP 1, another mixture of ethanol-acetonitrile-acetic acid-triethylamine (80/20/0.2/0.3, $\mathrm{v} / \mathrm{v} / \mathrm{v} / \mathrm{v}$ ) was most widely applied as a mobile phase [23]. From these results, a certain mixture of ethanol or methanol in acetonitrile containing a small amount of acidic (acetic or trifluoroacetic acid) and basic (triethylamine) modifier is expected to be used as a mobile phase for the resolution of fendiline and its analogues on CSP 1. To find out the most widely applicable mobile phase condition for the resolution of fendiline and its analogues, we selected five analytes including fendiline and its analogues 8, 12, 13 and 17. Analytes 8 and 12 were selected to represent analogues $(\mathbf{8}-\mathbf{1 2})$ containing ortho-substituted phenyl or 1-naphthyl group and analytes 13 and 17 were selected to represent analogues (13-17) containing para-substituted phenyl or 2-naphthyl group instead of the simple phenyl group at the chiral center of fendiline. The selected five analytes were resolved on CSP 1 with the variation of the mobile phase composition. The resolutions of the selected five analytes on CSP $\mathbf{1}$ with the variation of the type and content of alcohol in acetonitrile at the constant ratio of trifluoroacetic acid-triethylamine 
$(0.1 / 0.5, \mathrm{v} / \mathrm{v})$ are summarized in Table 1 . The retention factors $\left(k_{1}\right)$ for the resolution of selected five analytes were found to increase as the content of methanol in acetonitrile was increased from $50 \%$ to $80 \%$. In addition, the retention factors $\left(k_{1}\right)$ for the resolution of selected five analytes were also found to increase as the type of alcohol in acetonitrile was changed from methanol to ethanol and then to 2-propanol. By increasing the content of methanol in acetonitrile, the mobile phase polarity seems to decrease. By changing the type of alcohol in acetonitrile from methanol to ethanol and then to 2-propanol, the mobile phase polarity is also expected to decrease continuously. When the mobile phase polarity is decreased, the interaction between the mobile phase and analytes is diminished and, consequently, the retention factors $\left(k_{1}\right)$ increase. However, the separation factors $(\alpha)$ and resolutions $\left(\mathrm{R}_{\mathrm{S}}\right)$ for the resolution of selected five analytes on CSP 1 did not show any significant trends. For fendiline, 12 and 13, 80\% methanol in acetonitrile was found to show the best resolution results especially in terms of the resolutions ( $\left.\mathrm{R}_{\mathrm{S}}\right)$. However, for the resolution of $\mathbf{8}$ and 17, the best resolution results in terms of both the separation factors $(\alpha)$ and resolutions $\left(\mathrm{Rs}_{\mathrm{s}}\right)$ were obtained when $80 \%$ ethanol in acetonitrile or $50 \%$ methanol in acetonitrile, respectively, was used.

As an effort to find out the optimum content of acidic and/or basic modifier in the mobile phase, the five analytes were resolved on CSP 1 with the variation of the ratio of trifluoroacetic acid and triethylamine in $80 \%$ methanol in acetonitrile and the resolution results are summarized in Table 2. As the content of trifluoroacetic acid is increased from $0.05 \%$ to $0.1 \%$ and then $0.2 \%$ (entry a, b and e) with a constant content of triethylamine $(0.5 \%)$, the retention factors $\left(k_{1}\right)$ increased continuously. However, the retention factors $\left(k_{1}\right)$ increased continuously as the content of triethylamine is decreased from $0.75 \%$ to $0.5 \%$ and then to $0.25 \%$ (entry $\mathrm{d}, \mathrm{b}$ and $\mathrm{c}$ ) with a constant content of trifluoroacetic acid $(0.1 \%)$. The retention factors $\left(k_{1}\right)$ also increased as the content of triethylamine is decreased from $0.75 \%$ and then to $0.50 \%$ (entry $f$ and e) with a constant content of trifluoroacetic acid $(0.20 \%)$. In general, the retention factors $\left(k_{1}\right)$ increase as the content of trifluoroacetic acid is increased and as the content of triethylamine is decreased. As the content of trifluoroacetic acid is increased or as the content of triethylamine is decreased in the mobile phase, the protonation state of the analyte amino group is expected to increase. In this instance, the interaction between the protonated amino group of analytes and the CSP increases and, consequently, the retention factors are expected to increase as the content of trifluoroacetic acid is increased or as the content of triethylamine is decreased in the mobile phase.

While the retention factors $\left(k_{1}\right)$ show some trends with the variation of the ratio of trifluoroacetic acid and triethylamine in $80 \%$ methanol in acetonitrile as shown in Table 2, the separation factors $(\alpha)$ were found not to show any specific trend. The separation factors $(\alpha)$ were found to vary only in small range with the variation of the ratio of trifluoroacetic acid and triethylamine in the mobile phase. The resolutions (Rs) were also found not to show any specific trend. When the ratio of trifluoroacetic acid and triethylamine was $0.10 / 0.25(\mathrm{v} / \mathrm{v}$, entry $\mathrm{c})$, the retention factors $\left(k_{1}\right)$ were highest, but the separation factors $(\alpha)$ and resolutions (Rs) were worst. For the resolution of fendiline, the baseline resolution ( $\mathrm{Rs}$ is $>1.5$ ) was obtained only with the use of a mobile phase consisting of methanol-acetonitrile-trifluoroacetic acid-triethylamine at a ratio of 80/20/0.1/0.5 (v/v/v/v) (entry b in Table 2). 
Table 1. Resolution of racemic fendiline and its analogues $(\mathbf{8}, \mathbf{1 2}, \mathbf{1 3}$ and 17) on CSP 1 with the variation of the content of methanol (MeOH), ethanol (EtOH) or 2-propanol (PrOH) in acetonitrile (ACN) as a mobile phase containing trifluoroacetic acid (TFA)-triethylamine (TEA) of the constant ratio $[\mathrm{MeOH}$ or EtOH or iPrOH-ACN-TFA-TEA, $\mathrm{x} /(100-\mathrm{x}) / 0.1 / 0.5, \mathrm{v} / \mathrm{v} / \mathrm{v} / \mathrm{v}]$. Flow rate: $0.5 \mathrm{~mL} / \mathrm{min}$. Detection: $254 \mathrm{~nm} \mathrm{UV}$. Column temperature: $20^{\circ} \mathrm{C}$. $k_{1}$ : Retention factor of the first eluted enantiomer. $\alpha$ : Separation factor. Rs: Resolution.

\begin{tabular}{|c|c|c|c|c|c|c|c|c|c|c|c|c|c|c|c|}
\hline \multirow{2}{*}{ Alcohol Content (x) } & \multicolumn{3}{|c|}{ Fendiline } & \multicolumn{3}{|c|}{8} & \multicolumn{3}{|c|}{12} & \multicolumn{3}{|c|}{13} & \multicolumn{3}{|c|}{17} \\
\hline & $k_{1}$ & $\alpha$ & $\mathbf{R}_{\mathrm{S}}$ & $k_{1}$ & $\alpha$ & $\mathbf{R}_{\mathrm{S}}$ & $k_{1}$ & $\alpha$ & $\mathbf{R}_{\mathbf{S}}$ & $k_{1}$ & $\alpha$ & $\mathbf{R}_{\mathbf{S}}$ & $k_{1}$ & $\alpha$ & $\mathbf{R}_{\mathrm{S}}$ \\
\hline $50 \% \mathrm{MeOH}$ & 1.21 & 1.22 & 0.92 & 1.13 & 1.24 & 0.97 & 1.28 & 1.35 & 1.48 & 1.13 & 1.24 & 0.97 & 1.28 & 1.35 & 1.48 \\
\hline $80 \% \mathrm{MeOH}$ & 1.91 & 1.25 & 1.55 & 2.36 & 1.25 & 1.31 & 2.08 & 1.28 & 1.15 & 2.36 & 1.25 & 1.31 & 2.08 & 1.28 & 1.15 \\
\hline $80 \% \mathrm{EtOH}$ & 3.20 & 1.27 & 1.09 & 4.06 & 1.27 & 1.13 & 3.12 & 1.31 & 0.89 & 4.06 & 1.27 & 1.13 & 3.12 & 1.31 & 0.89 \\
\hline $80 \% \mathrm{PrOH}$ & 4.49 & 1.23 & 0.76 & 5.64 & 1.23 & 0.59 & 5.25 & 1.26 & 1.01 & 5.64 & 1.23 & 0.59 & 5.25 & 1.26 & 1.01 \\
\hline
\end{tabular}

Table 2. Resolution of racemic fendiline and its analogues $(\mathbf{8}, \mathbf{1 2}, \mathbf{1 3}$ and 17) on CSP $\mathbf{1}$ with the variation of the ratio of trifluoroacetic acetic acid (TFA)-triethylamine (TEA) in 80\% methanol in acetonitrile as a mobile phase (MeOH-ACN-TFA-TEA, 80/20/x/y, v/v/v/v). Flow rate: $0.5 \mathrm{~mL} / \mathrm{min}$. Detection: $254 \mathrm{~nm}$ UV. Column temperature: $20^{\circ} \mathrm{C}$. $k_{1}$ : Retention factor of the first eluted enantiomer. $\alpha$ : Separation factor. Rs: Resolution.

\begin{tabular}{|c|c|c|c|c|c|c|c|c|c|c|c|c|c|c|c|c|}
\hline \multirow{2}{*}{ Entry } & \multirow{2}{*}{ TFA/TEA Ratio (v/v) } & \multicolumn{3}{|c|}{ Fendiline } & \multicolumn{3}{|c|}{8} & \multicolumn{3}{|c|}{12} & \multicolumn{3}{|c|}{13} & \multicolumn{3}{|c|}{17} \\
\hline & & $k_{1}$ & $\alpha$ & $\mathbf{R}_{\mathbf{S}}$ & $k_{1}$ & $\alpha$ & $\mathbf{R}_{\mathbf{S}}$ & $k_{1}$ & $\alpha$ & $\mathbf{R}_{\mathbf{S}}$ & $k_{1}$ & $\alpha$ & $\mathbf{R}_{\mathbf{S}}$ & $k_{1}$ & $\alpha$ & $\mathbf{R}_{\mathrm{S}}$ \\
\hline $\mathrm{a}$ & $0.05 / 0.5$ & 1.57 & 1.27 & 1.19 & 1.81 & 1.41 & 1.60 & 1.61 & 1.50 & 2.56 & 2.08 & 1.25 & 1.16 & 1.88 & 1.29 & 1.52 \\
\hline $\mathrm{b}$ & $0.1 / 0.5$ & 1.91 & 1.25 & 1.55 & 1.98 & 1.39 & 1.24 & 1.82 & 1.54 & 2.50 & 2.36 & 1.25 & 1.31 & 2.08 & 1.28 & 1.15 \\
\hline $\mathrm{c}$ & $0.1 / 0.25$ & 4.30 & 1.10 & 0.48 & 4.37 & 1.23 & 0.88 & 4.40 & 1.33 & 1.73 & 5.25 & 1.13 & 0.83 & 4.75 & 1.15 & 0.74 \\
\hline $\mathrm{d}$ & $0.1 / 0.75$ & 1.16 & 1.21 & 0.78 & 1.39 & 1.37 & 1.38 & 1.23 & 1.47 & 2.11 & 1.69 & 1.22 & 1.19 & 1.46 & 1.25 & 1.42 \\
\hline $\mathrm{e}$ & $0.2 / 0.5$ & 2.55 & 1.25 & 1.20 & 3.03 & 1.40 & 1.41 & 2.87 & 1.48 & 1.92 & 3.15 & 1.24 & 1.09 & 3.15 & 1.28 & 1.33 \\
\hline f & $0.2 / 0.75$ & 1.49 & 1.19 & 0.84 & 1.49 & 1.32 & 1.01 & 1.47 & 1.45 & 1.85 & 2.05 & 1.21 & 1.47 & 1.66 & 1.23 & 0.98 \\
\hline
\end{tabular}


Resolution of analytes $\mathbf{1 2}$ and $\mathbf{1 3}$ was also quite good with the use of a mobile phase consisting of methanol-acetonitrile-trifluoroacetic acid-triethylamine at a ratio of 80/20/0.1/0.5 (v/v/v/v). However, a mobile phase consisting of methanol-acetonitrile-trifluoroacetic acid-triethylamine at a ratio of $80 / 20 / 0.05 / 0.5(\mathrm{v} / \mathrm{v} / \mathrm{v} / \mathrm{v})$ (entry a in Table 2) was found to be the best mobile phase condition for the resolution of analytes $\mathbf{8}$ and $\mathbf{1 7}$ in terms of both the separation factors and resolutions. As an acidic modifier, acetic acid was also tested instead of trifluoroacetic acid. However, the mobile phase containing acetic acid was found to be inferior to that containing trifluoroacetic acid. For example, fendiline was resolved with the separation factor $(\alpha)$ of 1.25 and resolution (Rs) of 1.55 when a mobile phase consisting of methanol-acetonitrile-trifluoroacetic acid-triethylamine at a ratio of 80/20/0.1/0.5 ( $/ \mathrm{v} / \mathrm{v} / \mathrm{v} / \mathrm{v})$ was used, but it was resolved with the separation factor $(\alpha)$ of 1.24 and resolution ( $\left.\mathrm{R}_{\mathrm{S}}\right)$ of 1.13 when a mobile phase consisting of methanol-acetonitrile-acetic acid-triethylamine at a ratio of 80/20/0.1/0.5 $(\mathrm{v} / \mathrm{v} / \mathrm{v} / \mathrm{v})$ was used.

Column temperature is also an important factor for the resolution of racemic primary and secondary amino compounds on CSP 1 [12]. For the resolution of racemic compounds containing a primary amino group on CSP 1, the separation factors $(\alpha)$ have been reported to increase always as the column temperature was decreased as usual [12]. However, for the resolution of $\beta$-blockers containing a secondary amino group on CSP 1, the separation factors $(\alpha)$ were found, very surprisingly and unusually, to increase as the column temperature was increased [20]. Based on the van't Hoff plots, both $\Delta \Delta \mathrm{H}$ and $\Delta \Delta \mathrm{S}$ values for the resolution of $\beta$-blockers on CSP 1 were calculated to be positive and, consequently, the negative $\Delta \Delta \mathrm{G}$ values corresponding to the separation factor $(\alpha)$ of greater than 1.00 were concluded to be entirely dependent on the $\Delta \Delta \mathrm{S}$ values, indicating that the resolution of $\beta$-blockers on CSP $\mathbf{1}$ is entropy controlled [20]. Fendiline is a secondary amino compound. In this instance, the resolution of fendiline on CSP 1 is expected to follow the unusual temperature effect on the chiral recognition. However, when the column temperature was changed from 30 to 20 and then to $10{ }^{\circ} \mathrm{C}$ for the resolution of fendiline on CSP 1, the separation factor $(\alpha)$ increased continuously from 1.21 to 1.25 and then to 1.32 . These results indicate that the resolution of fendiline on CSP 1 follows the usual temperature effect on the chiral recognition.

In order to compare the chromatographic behavior for the resolution of fendiline with those for the resolution of its analogues (2-17) on CSP 1, all analytes were resolved with the use of a mobile phase consisting of methanol-acetonitrile-trifluoroacetic acid-triethylamine at a ratio of 80/20/0.1/0.5 $(\mathrm{v} / \mathrm{v} / \mathrm{v} / \mathrm{v})$, the best mobile phase condition for the resolution of fendiline, and the resolution results are summarized in Table 3 and the representative chromatograms are illustrated in Figure 3 . The elution orders for fendiline and its analogues 4, 6, 12 and 17 shown in Table 3 were determined by injecting configurationally known optically active samples, which were prepared from the commercially available optically active 1-phenylethylamine, 1-( $\alpha$-naphthyl)ethylamine or 1-( $\beta$-naphthyl)ethylamine. For other analytes, the elution orders were not determined because the elution orders were not clear (for analogues $\mathbf{3}$ and 5) or optically active samples were not able to be prepared due to the lack of optically active starting materials (for analogues 7-11 and 13-16).

Interestingly, the baseline resolution of fendiline makes CSP 1 useful for the determination of the enantiomeric purity or enantiomeric composition of biologically more active $(R)$-fendiline. The comparison of the chromatograms shown in Figure 4 for the resolution of racemic fendiline and optically active $(R)$-fendiline prepared from $(R)$-phenylethylamine of more than $99 \%$ ee demonstrates the usefulness of 
CSP 1 for the determination of enantiomeric purity of $(R)$-fendiline. From Figure 4, the enantiomeric purity of the $(R)$-fendiline is concluded to be more than $99 \%$ ee because the peak corresponding to the $(S)$-enantiomer is not shown in the chromatogram obtained from the sample of $(R)$-fendiline.

Figure 3. Representative chromatograms for the resolution of (a) fendiline and its analogues; (b) 10; (c) 12; and (d) 13 with the use of a mobile phase consisting of methanol-acetonitrile-trifluoroacetic acid-triethylamine at a ratio of 80/20/0/1/0.5 (v/v/v/v). Flow rate: $0.5 \mathrm{~mL} / \mathrm{min}$. Detection: $254 \mathrm{~nm} \mathrm{UV}$. Temperature, $20^{\circ} \mathrm{C}$.
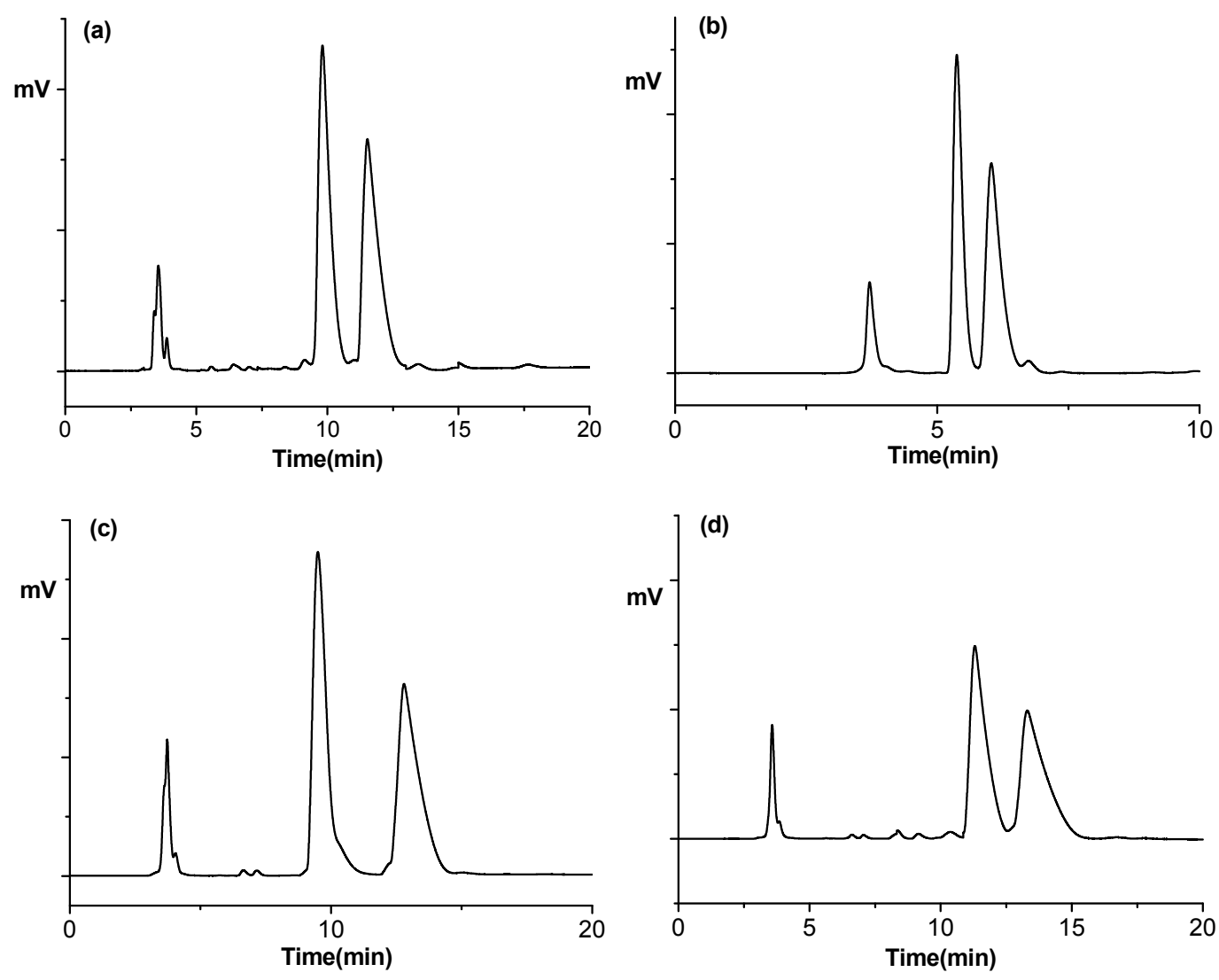

Figure 4. Comparison of the chromatograms for the resolution of (a) racemic fendiline and (b) $(R)$-fendiline on CSP 1 with the use of a mobile phase consisting of methanol-acetonitrile-trifluoroacetic acid-triethylamine at a ratio of 80/20/0.1/0.5 (v/v/v/v). Flow rate: $0.5 \mathrm{~mL} / \mathrm{min}$. Detection: $254 \mathrm{~nm} \mathrm{UV}$. Temperature, $20^{\circ} \mathrm{C}$.

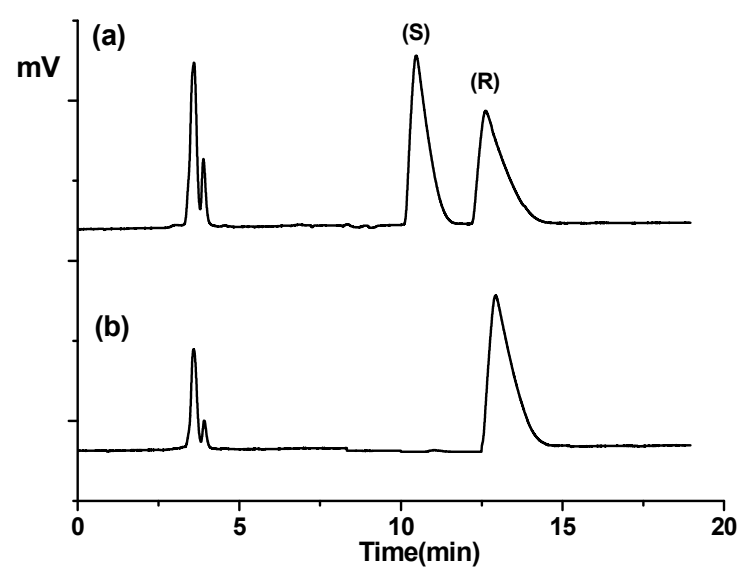


Table 3. Resolution of racemic fendiline and its analogues (2-17) on CSP 1 with the use of a mixture of methanol-acetonitrile-trifluoroacetic acid-triethylamine (MeOH-ACN-TFATEA, 80/20/0.1/0.5, v/v/v/v) as a mobile phase. Flow rate: $0.5 \mathrm{~mL} / \mathrm{min}$. Detection: $254 \mathrm{~nm}$ UV. Temperature, $20^{\circ} \mathrm{C} ; k_{1}$, retention factor of the first eluted enantiomer; $k_{2}$, retention factor of the second eluted enantiomer. Absolute configurations of the first and second eluted enantiomers are indicated in the parenthesis. $\alpha$, separation factor; Rs, resolution.

\begin{tabular}{ccccc}
\hline Analytes & $\boldsymbol{k}_{\mathbf{1}}$ & $\boldsymbol{k}_{\mathbf{2}}$ & $\boldsymbol{\alpha}$ & $\mathbf{R}_{\mathbf{S}}$ \\
\hline fendiline & $1.91(\mathrm{~S})$ & $2.42(\mathrm{R})$ & 1.25 & 1.55 \\
$\mathbf{2}$ & 1.56 & 1.76 & 1.13 & 0.42 \\
$\mathbf{3}$ & 0.95 & 1.03 & 1.08 & 0.48 \\
$\mathbf{4}$ & $1.61(\mathrm{~S})$ & $1.85(\mathrm{R})$ & 1.15 & 0.74 \\
$\mathbf{5}$ & 1.33 & 1.50 & 1.13 & 0.58 \\
$\mathbf{6}$ & $0.92(\mathrm{~S})$ & $1.04(\mathrm{R})$ & 1.12 & 0.74 \\
$\mathbf{7}$ & 1.64 & 2.14 & 1.31 & 0.87 \\
$\mathbf{8}$ & 1.98 & 2.76 & 1.39 & 1.24 \\
$\mathbf{9}$ & 0.69 & 0.89 & 1.28 & 1.27 \\
$\mathbf{1 0}$ & 0.59 & 0.79 & 1.33 & 1.42 \\
$\mathbf{1 1}$ & 2.39 & 3.02 & 1.26 & 1.23 \\
$\mathbf{1 2}$ & $1.82(\mathrm{~S})$ & $2.80(\mathrm{R})$ & 1.54 & 2.50 \\
$\mathbf{1 3}$ & 2.36 & 2.95 & 1.25 & 1.31 \\
$\mathbf{1 4}$ & 1.57 & 1.92 & 1.22 & 1.12 \\
$\mathbf{1 5}$ & 1.61 & 1.97 & 1.22 & 0.79 \\
$\mathbf{1 6}$ & 3.10 & 3.77 & 1.22 & 0.97 \\
$\mathbf{1 7}$ & $2.08(\mathrm{~S})$ & $2.65(\mathrm{R})$ & 1.28 & 1.15 \\
\hline
\end{tabular}

When the 3,3-diphenylpropyl group at the secondary nitrogen atom of fendiline was replaced with simple $n$-alkyl, sterically bulky alkyl or 2-phenylalkyl group (analytes 2-6), both of the separation factors and resolutions were decreased quite much. Consequently, the 3,3-diphenylpropyl group bonded to the secondary amino group of fendiline is expected to play an important role in the chiral recognition. When the methyl group at the chiral center of fendiline was changed as a longer alkyl group (analyte 7), both of the separation factors and resolutions were also decreased quite much, indicating the importance of the methyl group at the chiral center in the chiral recognition. By changing the methyl group as a longer alkyl group at the chiral center of fendiline, the size difference between the two groups at the chiral center of analyte is expected to be diminished and, consequently, the discrimination of the two enantiomers might become less effective. When the phenyl group at the chiral center of fendiline was replaced with ortho-substituted phenyl group (analytes 8-11) or 1-naphthyl group (analyte 12), the separation factors increased, but the resolutions decreased compared with that for fendiline except for analyte 12. However, the phenyl group at the chiral center of fendiline was replaced with para-substituted phenyl group (analytes 13-16) or 2-naphthyl group (analyte 17), the separation factors were not changed much, but the resolutions decreased in every case compared with that of fendiline. By replacing the phenyl group at the chiral center of fendiline with an ortho-substituted phenyl group, the steric bulkiness of the substituent experienced at the chiral center of analytes is expected to increase and, consequently, the size difference between the two groups at the chiral center of analyte is expected to increase and, consequently, the discrimination of the two enantiomers might become more effective. The steric bulkiness experienced at the chiral center of analytes with the ortho-substituted phenyl group seems to be greater than that experienced with the para-substituted 
phenyl group. In this instance, an analyte containing an ortho-substituted phenyl group should show greater chiral recognition than the corresponding analyte containing a para-substituted phenyl group. In particular, analyte 12 containing 1-naphthyl group at the chiral center was resolved much better than fendiline. The 1-naphthyl group at the chiral center of analyte 12 seems to be much bulkier group than the ortho- or para-substituted phenyl group or 2-naphthyl group and, consequently, analyte $\mathbf{1 2}$ is expected to be resolved best. However, the exact chiral recognition mechanism is not clear yet.

\section{Experimental Section}

\subsection{Chromatography}

Liquid chromatographic results for the resolution of fendiline and its analogues were obtained with an HPLC system consisting of a Rheodyne model 7725i injector (Rohnert Park, CA, USA) with a $20 \mu \mathrm{L}$ sample loop, a Waters model 515 HPLC pump (Milford, MA, USA), a Waters 484 Tunable Absorbance detector and a YoungLin Autochro data module (Software: YoungLin Autochro-WIN 2.0 plus). The chiral column temperature was maintained at $20{ }^{\circ} \mathrm{C}$ by using a JEIO TECH VTRC-620 cooling circulator (Daejeon, Korea). Chiral column packed with end-capped CSP 1 [Chirosil RCA $(+)$, $150 \mathrm{~mm} \times 4.6 \mathrm{~mm}$ I.D.] was available from RS tech (Daejeon, Korea). The number of theoretical plates $(\mathrm{N})$ of the chiral column calculated from the retention time and the peak width at half height for the first eluted enantiomer of fendiline was 2537. Injection samples were prepared by dissolving each analyte in chloroform at a concentration of $1.0 \mathrm{mg} / \mathrm{mL}$. The injection volume was usually $1.0 \mu \mathrm{L}$.

\subsection{Preparation of Fendiline and Its Analogues}

Fendiline and its analogues were prepared according to the scheme shown in Figure 5. Starting materials for the preparation of fendiline and its analogues 2-6, 12 and 17 including 1-phenylethylamine $\left(\mathrm{Ar}=\right.$ phenyl, $\mathrm{R}_{1}=\mathrm{CH}_{3}$, compound $\mathrm{B}$ in Figure 5), 1-( $\alpha$-naphthyl)ethylamine ( $\mathrm{Ar}=\alpha$-naphthyl, $\mathrm{R}_{1}=\mathrm{CH}_{3}$, compound $\mathrm{B}$ in Figure 5) and 1-( $\beta$-naphthyl)ethylamine $\left(\mathrm{Ar}=\beta\right.$-naphthyl, $\mathrm{R}_{1}=\mathrm{CH}_{3}$, compound B in Figure 5) were commercially available from Sigma-Aldrich (St. Louis, MO, USA). Starting materials for the preparation of fendiline analogues $\mathbf{7 - 1 1}$ and $\mathbf{1 3 - 1 6}$ such as 1-(substituted phenyl)ethylamines and 1-phenylbutylamine were prepared through the reductive amination of appropriate aryl alkyl ketones (compound A in Figure 5) by treating aryl alkyl ketones with sodium cyanoborohydride $\left(\mathrm{NaCNBH}_{3}\right)$ and ammonium acetate $\left(\mathrm{NH}_{4} \mathrm{OAc}\right)$ in methanol. Compound $\mathrm{B}$ then was treated with carboxylic acid in the presence of 1-ethyl-3-(3-dimethylaminopropyl)carbodiimide hydrochloride (EDC $\cdot \mathrm{HCl}$, carboxylic acid group activating reagent) and $N, N$-diisopropylethylamine (DIEA) in methylene chloride or treated with acid chloride in the presence of triethylamine in methylene chloride. Then the resulting amides were treated with $\mathrm{LiAlH}_{4}$ in tetrahydrofuran (THF) to afford fendiline and its analogues. The structures of fendiline and its analogues (2-17) were identified by ${ }^{1} \mathrm{H}-\mathrm{NMR}$ spectra. As an example, the detailed synthetic procedure for the preparation of fendiline analogue $\mathbf{8}$ is provided as follows.

1-(2-Methylphenyl)ethanone $(0.50 \mathrm{~mL}, 3.73 \mathrm{mmol})$ and ammonium acetate $(2.88 \mathrm{~g}, 37.3 \mathrm{mmol})$ were dissolved in methanol $(20 \mathrm{~mL})$. To the stirred solution was added slowly a solution of $\mathrm{NaCNBH}_{3}$ $(0.79 \mathrm{~g}, 11.2 \mathrm{mmol})$ dissolved in methanol $(15 \mathrm{~mL})$ through a dropping funnel. The whole mixture was 
stirred at room temperature. After stirring for $12 \mathrm{~h}$, solvent was removed by using rotary evaporator. The residue was dissolved in ethyl acetate and then the solution was treated with $6 \mathrm{~N} \mathrm{HCl}$ solution to make the solution to be acidic $(\mathrm{pH}=12-13)$. The two layers were separated and then $6 \mathrm{~N} \mathrm{NaOH}$ solution was added to the separated aqueous solution to make the solution to be basic $(\mathrm{pH}=11-12)$. The basic aqueous solution was extracted with ethyl acetate twice and then combined organic solution was dried over anhydrous $\mathrm{Na}_{2} \mathrm{SO}_{4}$. Solvent was removed by using rotary evaporator to afford an intermediate amine $\mathrm{B}, 1$-(2-methylphenyl)ethylamine ( $\mathrm{Ar}=$ 2-methylphenyl, $\mathrm{R}_{1}=\mathrm{CH}_{3}$, compound $\mathrm{B}$ in Figure 5) $(0.21 \mathrm{~g}, 46 \%$ yield $)$.

Figure 5. Scheme for the preparation of fendiline and its analogues (2-17).

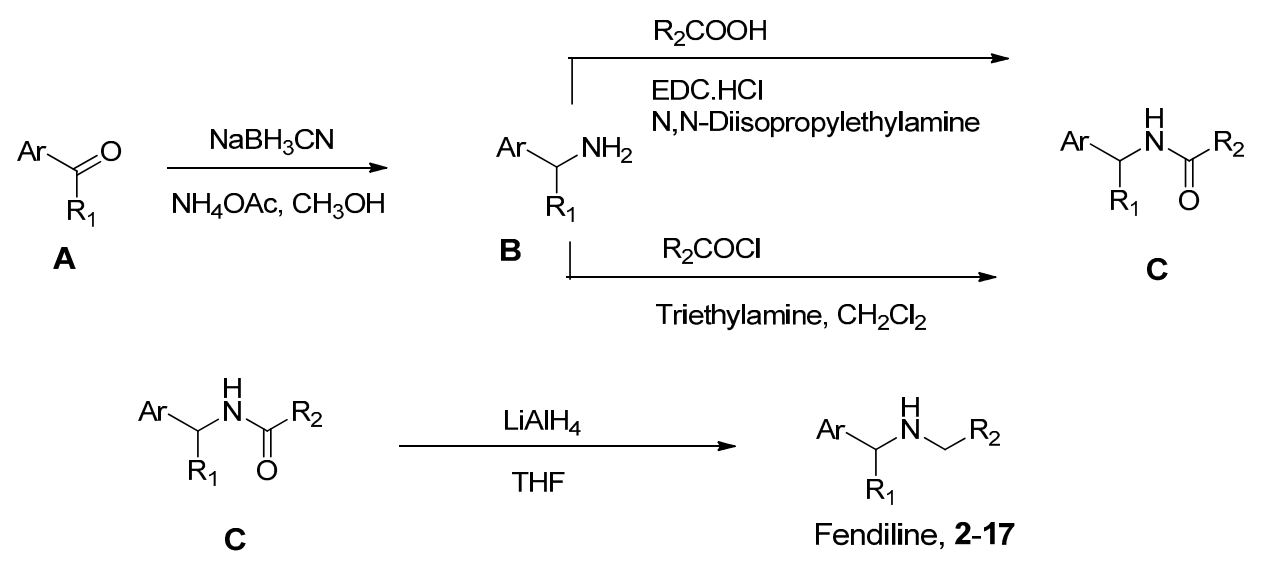

3,3-Diphenylpropionic acid (1.43 g, $10.5 \mathrm{mmol})$ was dissolved in methylene chloride $(50 \mathrm{~mL})$. To the solution was added EDC.HCl (2.22 g, $11.6 \mathrm{mmol})$ and DIEA (1.09 $\mathrm{mL}, 11.6 \mathrm{mmol})$. The mixture was stirred for $30 \mathrm{~min}$ at room temperature under an argon atmosphere. To the stirred solution was added 1-(2-methylphenyl)ethylamine (1.56 g, $16.6 \mathrm{mmol})$. After stirring for $12 \mathrm{~h}$ at room temperature, the whole mixture solution was treated with water $(50 \mathrm{~mL})$. The separated organic solution was dried over anhydrous $\mathrm{Na}_{2} \mathrm{SO}_{4}$. Solvent was removed by using rotary evaporator. The residue was purified by silica gel column chromatography (hexane/ethyl acetate, 95/5, v/v) to afford an intermediate amide C, 3,3-diphenyl- $N$-(1-(o-tolyl)ethyl)propanamide (Ar = 2-methylphenyl, $\mathrm{R}_{1}=\mathrm{CH}_{3}, \mathrm{R}_{2}=$ 2,2-diphenylethyl, compound C in Figure 5) (1.0 g, 27.8\% yield).

$\mathrm{LiAlH}_{4}(0.40 \mathrm{~g})$ was dissolved in THF $(20 \mathrm{~mL})$. To the solution was added a solution of 3,3-diphenyl- $N$-(1-(o-tolyl)ethyl)propanamide $(0.45 \mathrm{~g}, 1.31 \mathrm{mmol})$ dissolved in THF $(10 \mathrm{~mL})$ through a dropping funnel with stirring at $0{ }^{\circ} \mathrm{C}$. And then the whole mixture was heated to reflux for $16 \mathrm{~h}$. After removing solvent by using a rotary evaporator, the residue was dissolved in ethyl acetate. The ethyl acetate solution was washed with water. The organic solution was dried over anhydrous $\mathrm{Na}_{2} \mathrm{SO}_{4}$. Solvent was removed by using rotary evaporator. The residue was purified by silica gel column chromatography to afford fendiline analogue $8(0.11 \mathrm{~g}, 40 \%$ yield $) .{ }^{1} \mathrm{H}-\mathrm{NMR}\left(\mathrm{CDCl}_{3}, 300 \mathrm{MHz}\right)$ d 7.37-7.11 (m, 14H), 4.03-3.94 (m, 2H), 2.52-2.41 (m, 2H), 2.29-2.20 (m, 5H), $1.26(\mathrm{~d}, 3 \mathrm{H})$.

\section{Conclusions}

Fendiline and its sixteen analogues were resolved on CSP 1. The separation factor $(\alpha)$ and resolution (Rs) for fendiline were found to be 1.25 and 1.55 , respectively, with the use of a mobile 
phase consisting of methanol-acetonitrile-trifluoroacetic acid-triethylamine at a ratio of 80/20/0.1/0.5 $(\mathrm{v} / \mathrm{v} / \mathrm{v} / \mathrm{v})$. In order to elucidate the structural characteristics required for the resolution of fendiline on CSP 1, the chromatographic resolution behaviors for fendiline and its analogues were compared. The 3,3-diphenylpropyl group of fendiline and the difference in the steric bulkiness between the phenyl group and the methyl group at the chiral center of fendiline were found to be important for the chiral recognition. However, the exact chiral recognition mechanism needs further study.

\section{Acknowledgments}

This work was supported by the BK21 Plus program, National Research Foundation (NRF), Korea.

\section{Author Contributions}

G.R.L. performed the analyte synthesis, chromatographic data collection and data analysis. M.H.H. designed and supervised the study.

\section{Conflicts of Interest}

The authors declare no conflict of interest.

\section{References}

1. Bayer, R.; Mannhold, R. Fendiline: A review of its basic pharmacological and clinical properties. Pharmatherapeutica 1987, 5, 103-136.

2. Nguyen, L.A.; He, H.; Pham-Huy, C. Chiral drugs: An overview. Int. J. Biomed. Sci. 2006, 2, 85-100.

3. Wilkinson, J.; Foretia, D.; Rossington, S.; Anthony Heagerty, A.; Leonard, J.; Hussain, N.; Austin, C. 2'-Hydroxy-fendiline analogues as potent relaxers of isolated arteries. Eur. J. Pharmacol. 2007, 561, $160-163$.

4. Zhang, Y.; Wu, D.-R.; Wang-Iverson, D.B.; Tymiak, A.A. Enantioselective chromatography in drug discovery. Drug Dis. Today 2005, 10, 571-577.

5. Cavazzini, A.; Pasti, L.; Massi, A.; Marchetti, N.; Dondi, F. Recent applications in chiral high performance liquid chromatography: A review. Anal. Chim. Acta 2011, 706, 205-222.

6. Tang, M.; Zhang, J.; Zhuang, S.; Liu, W. Development of chiral stationary phases for high-performance liquid chromatographic separation. TrAC Trends Anal. Chem. 2012, 39, 180-194.

7. Chankvetadze, B. Recent developments on polysaccharide-based chiral stationary phases for liquid-phase separation of enantiomers. J. Chromatogr. A 2012, 1269, $26-51$.

8. Xiao, Y.; Ng, S.-C.; Tan, T.T.Y.; Wang, Y. Recent development of cyclodextrin chiral stationary phases and their applications in chromatography. J. Chromatogr. A 2012, 1269, 52-68.

9. Ward, T.J.; Faris, A.B., III. Chiral separations using the macrocyclic antibiotics: A review. J. Chromatogr. A 2001, 906, 73-89.

10. D’Acquarica, I.; Gasparrini, F.; Misiti, D.; Pierini, M.; Villani, C. HPLC chiral stationary phases containing macrocyclic antibiotics: Practical aspects and recognition mechanism. In Advances in Chromatography; Grushka, E., Grinberg, N., Eds.; CRC Press: Boca Raton, FL, USA, 2007; Volume 46, pp. 109-173. 
11. Sun, P.; Armstrong, D. Effective enantiomeric separations of racemic primary amines by the isopropyl carbamate-cyclofructan6 chiral stationary phase. J. Chromatogr. A 2010, 1217, 4904-4918.

12. Choi, H.J.; Hyun, M.H. Liquid chromatographic chiral separations by crown ether-based chiral stationary phases. J. Liquid Chromatogr. Relat. Technol. 2007, 30, 853-875.

13. Ema, T. Synthetic macrocyclic receptors in chiral analysis and separation. J. Incl. Phenom. Macrocycl. Chem. 2012, 74, 41-55.

14. Németh, T.; Lévai, S.; Kormos, A.; Kupai, J.; Tõth, T.; Balogh, G.T.; Huszthy, P. Preparation and studies of chiral stationary phases containing enantiopure acridino-18-crown-6 ether selectors. Chirality 2014, 26, 651-654.

15. Gasparrini, F.; Misiti, D.; Pierini, M.; Villani, C. Enantioselective chromatography on brush-type chiral stationary phases containing totally synthetic selectors: Theoretical aspects and practical applications. J. Chromatogr. A 1996, 724, 79-90.

16. Hermansson, J.; Grahn, A. Optimization of the separation of enantiomers of basic drugs: Retention mechanisms and dynamic modification of the chiral bonding properties on an $\alpha_{1}$-acid glycoprotein column. J. Chromatogr. A 1995, 694, 57-69.

17. Hyun, M.H. Characterization of liquid chromatographic chiral separation on chiral crown ether stationary phases. J. Sep. Sci. 2003, 26, 242-250.

18. Hyun, M.H. Development and application of crown ether-based HPLC chiral stationary phases. Bull. Korean Chem. Soc. 2005, 26, 1153-1163.

19. Hyun, M.H. Preparation and application of HPLC chiral stationary phases based on (+)-(18-crown-6)-2,3,11,12-tetracarboxylic acid. J. Sep. Sci. 2006, 29, 750-761.

20. Zhang, D.; Li, F.; Kim, D.H.; Choi, H.J.; Hyun, M.H. Resolution of $\beta$-blockers on a chiral stationary phase based on (+)-(18-crown-6)-2,3,11,12-tetracarboxylic acid: Unusual temperature effect. J. Chromatogr. A 2005, 1083, 89-95.

21. Lee, A.; Choi, H.J.; Hyun, M.H. Liquid chromatographic direct resolution of flecainide and its analogs on a chiral stationary phase based on (1)-(18-crown-6)-2,3,11,12-tetracarboxylic acid. Chirality 2010, 22, 693-698.

22. Lee, A.; Choi, H.J.; Jin, K.B.; Hyun, M.H. Liquid chromatographic resolution of 1-aryl-1,2,3,4tetrahydroisoquinolines on a chiral stationary phase based on (+)-(18-crown-6)-2,3,11,12tetracarboxylic acid. J. Chromatogr. A 2011, 1218, 4071-4076.

23. Tak, K.M.; Park, E.J.; Hyun, M.H. Liquid chromatographic resolution of rasagiline and its analogues on a chiral stationary phase based on (+)-(18-crown-6)-2,3,11,12-tetracarboxylic acid. J. Sep. Sci. 2013, 36, 3682-3687.

Sample Availability: Samples of the compounds are available as chloroform solutions for HPLC injection.

(C) 2014 by the authors; licensee MDPI, Basel, Switzerland. This article is an open access article distributed under the terms and conditions of the Creative Commons Attribution license (http://creativecommons.org/licenses/by/4.0/). 